\title{
GMRES AND INTEGRAL OPERATORS *
}

\author{
C. T. KELLEY ${ }^{\dagger}$ AND Z. Q. XUE ${ }^{\dagger}$
}

\begin{abstract}
In this paper we show how the properties of integral operators and their approximations are reflected in the performance of the GMRES iteration and how these properties can be used to smooth the GMRES iterates, thereby strengthening the norm in which convergence takes place. The smoothed iteration has very similar properties to Broyden's method and we present some comparisons of the two methods with the standard (unsmoothed) implementation of GMRES.
\end{abstract}

Key words. Key words. Integral Equations, GMRES iteration, Broyden's Method

AMS(MOS) subject classifications. 65F10, 65J10, 65R20,

1. Introduction. In this paper we consider the performance of the GMRES [15] iteration for linear equations of the form

$$
A u=u-K u=f
$$

with $K$ a compact operator on a separable Hilbert space $H$. An example of such an operator is an integral operator on $H=L^{2}[0,1]$ of the form

$$
K u(x)=\int_{0}^{1} k(x, y) u(y) d y
$$

where $k$ is continuous.

Our setting is that of [7] where issues similar to those raised in this paper were considered in the context of Broyden's method [1] for linear and nonlinear equations. Broyden's method has also been considered as a linear equation solver in [2], [5], [6], and [10]. Let $H$ be a separable real Hilbert space and let $X \subset H$ be a Banach space such that the inner product $(\cdot, \cdot)$ in $H$ is continuous from $X \times X \rightarrow R$. This implies that there is $C_{X}$ such that

$$
\|u\|_{H} \leq C_{X}\|u\|_{X}
$$

for all $u \in X$. Let $K \in \mathcal{C O} \mathcal{M}(H, X)$ the space of compact operators from $H$ to $X$. Of course, we may also regard $K$ as an element of $\mathcal{C O M}(H)$ the space of compact operators on $H$. In the context of the integral operator (1.2) with continuous $k$, $H=L^{2}[0,1], X=C[0,1]$, and $C_{X}=1$.

Algorithms such as GMRES and Broyden's method, which depend upon notions of orthogonality could use the Hilbert space inner product of $H$ to solve equations in which the right hand side $f \in X$. However, a convergence theory based entirely on a Hilbert space formulation would show that the resulting sequence is convergent in the topology of $H$ but not necessarily in that of the Banach space $X$ in which the problem may have been originally posed. Hence, we face an apparent conflict between the topology in which the problem was posed and the inner product (and

* This document was printed on April 29, 1994.

$\dagger$ North Carolina State University, Center for Research in Scientific Computation and Department of Mathematics, Box 8205, Raleigh, N. C. 27695-8205, USA (Tim_Kelley@ncsu.edu, xue@math.ncsu.edu). This research was supported by National Science Foundation grants \#DMS9024622 and \#DMS-9321938. Computing activity was also partially supported by an allocation of time from the North Carolina Supercomputing Center. 
hence Hilbert space) nature of the algorithm. This issue was resolved in [7] in the context of Broyden's method. For the linear equations context of this paper the result of [7] is that the Broyden iterations converge q-superlinearly in the topology of $X$ provided $K \in \mathcal{C O M}(H, X)$ and $f \in X$. The purpose of this paper is to show how GMRES can be modified by an implicit Nyström interpolation [14] to have a similar property at a very small cost in both computational effort and algorithmic complexity.

Throughout this paper we assume that $A$ is a nonsingular linear operator on $H$ and on $X$. We consider convergence rate estimates of the form

$$
\left\|r_{k}\right\|_{H} \leq \tau_{k}\left\|r_{0}\right\|_{H}
$$

where the sequence of real numbers $\left\{\tau_{k}\right\}$ converges to zero and is independent of the right hand side $f$ of (1.1).

Rates of convergence of the form (1.4) can be derived from resolvent integration [11], [12] for any $K$ such that $I-K$ has bounded inverse and 1 is in the unbounded component of the resolvent of $K$. If $K$ is compact more precise information can be obtained, in fact the GMRES iterates converge r-superlinearly to the solution in a way that is independent of the right hand side. This means that the sequence $\left\{\tau_{k}\right\}$ converges q-superlinearly to zero $i$. $e$.

$$
\lim _{k \rightarrow \infty} \frac{\tau_{k+1}}{\tau_{k}}=0
$$

In the case of normal or diagonalizable (similar to normal) compact operators a q-superlinearly convergent sequence $\left\{\tau_{k}\right\}$ can be directly related to spectral properties of $K$ in a very simple way. In [9] orthogonal polynomials and assumptions on the rate of decay of the spectrum were used for this. In order to illustrate how the smoothing properties of $K$ might influence the convergence rate, we present a slight extension of the result in [9] below. While this result follows from the general theory in [12], we believe that its direct and brief proof is worth inclusion.

Theorem 1.1. Let $H$ be a separable Hilbert space, $K \in \mathcal{C O M}(H), A=I-K$ be nonsingular and let $S$ be a nonsingular bounded operator on $H$ such that

$$
L=S K S^{-1}
$$

is normal. Let $\left\{\lambda_{i}\right\}$ be the eigenvalues of $A$ ordered so that

$$
\left|\lambda_{i}-1\right| \geq\left|\lambda_{i+1}-1\right| \quad \text { for } i \geq 1
$$

then for all $k \geq 1$

$$
\left\|r_{k}\right\| \leq \kappa(S)\left\|r_{0}\right\| 2^{k} \prod_{i=1}^{k}\left|1-\lambda_{i}^{-1}\right| .
$$

Proof. For $k=1, \ldots$ define

$$
p_{k}(z)=\prod_{i=1}^{k}\left(1-\lambda_{i}^{-1} z\right) .
$$

Since $p_{k}(0)=1$ for all $k$ we have [15]

$$
\left\|r_{k}\right\|_{H} \leq \kappa(S)|| r_{0} \|_{H} \sup _{m}\left|p_{k}\left(\lambda_{m}\right)\right| .
$$


For $k$ fixed, $p_{k}\left(\lambda_{m}\right)=0$ for $1 \leq m \leq k$. For $m>k$

$$
\begin{aligned}
\left|p_{k}\left(\lambda_{m}\right)\right| & \leq \prod_{i=1}^{k}\left|1-\lambda_{i}^{-1} \lambda_{m}\right|=\prod_{i=1}^{k}\left|\lambda_{i}^{-1}\right|\left|\lambda_{i}-\lambda_{m}\right| \\
& \leq \prod_{i=1}^{k}\left|\lambda_{i}^{-1}\right|\left(\left|\lambda_{i}-1\right|+\left|1-\lambda_{m}\right|\right) \leq \prod_{i=1}^{k}\left|\lambda_{i}^{-1}\right| 2\left|\lambda_{i}-1\right| \\
& =2^{k} \prod_{i=1}^{k}\left|1-\lambda_{i}^{-1}\right| .
\end{aligned}
$$

This completes the proof. $\square$

Since $\lambda_{i} \rightarrow 1$, the sequence

$$
\tau_{k}=2^{k} \prod_{i=1}^{k}\left|1-\lambda_{i}^{-1}\right|
$$

is q-superlinearly convergent. If, say, $K$ is normal (so $S=I$ ) and the eigenfunctions of $K$ are smooth, then the rate of convergence of $\lambda_{i}$ to one reflects both the smoothness of the kernel $k$ and the convergence rate of the sequence $\left\{\tau_{k}\right\}$.

2. Convergence in a Stronger Norm. In this section we show how, given a rate estimate like (1.4) for the sequence of residuals, the GMRES iteration can be modified to produce a sequence that converges with the same rate in the norm of $X$.

Proposition 2.1. Let $\left\{u_{k}\right\}$ be the sequence of GMRES iterates. Assume that (1.4) holds for some sequence $\left\{\tau_{k}\right\}$. Let $\bar{u}_{k}=u_{k}+r_{k}$. Then

$$
\left\|\bar{u}_{k}-u^{*}\right\|_{X} \leq\|K\|_{\mathcal{L}(H, X)} \kappa_{H}(A) \tau_{k}\left\|u_{0}-u^{*}\right\|_{X} .
$$

Proof. First note that (1.4) implies that

$$
\left\|u_{k}-u^{*}\right\|_{H} \leq \tau_{k} \kappa_{H}(A)\left\|u_{0}-u^{*}\right\|_{H} .
$$

Since

$$
\bar{u}_{k}=u_{k}+r_{k}=f+K u_{k},
$$

continuity of $K$ as a map from $H$ to $X$ implies that

$$
\left\|\bar{u}_{k}-u^{*}\right\|_{X}=\left\|K\left(u_{k}-u^{*}\right)\right\|_{X} \leq\|K\|_{\mathcal{L}(H, X)}\left\|u_{k}-u^{*}\right\|_{H} .
$$

This completes the proof. $\square$

Note that $\bar{u}_{k}$ is as easy to compute as $u_{k}$ upon exit from the main loop in GMRES. An algorithmic description of GMRES is:

Algorithm 2.1. Algorithm gmres $(u, f, A, \epsilon)$

1. $r=f-A u, v_{1}=r /\|r\|_{H}, \rho=\|r\|_{H}, \beta=\rho, k=1$

2. While $\rho>\epsilon|| b \|_{H}$ do

(a) $v_{k+1}=A v_{k}$

for $j=1, \ldots k$

i. $h_{j k}=\left(v_{k+1}, v_{j}\right)$

ii. $v_{k+1}=v_{k+1}-h_{j k} v_{j}$

(b) $h_{k+1, k}=\left\|v_{k+1}\right\|_{H}$

(c) $v_{k+1}=v_{k+1} /\left\|v_{k+1}\right\|_{H}$ 
(d) $e_{1}=(1,0, \ldots, 0)^{T} \in R^{k+1}$

Minimize $\left\|\beta e_{1}-H_{k} y\right\|_{R^{k+1}}$ to obtain $y \in R^{k}$.

(e) $\rho=\left\|\beta e_{1}-H_{k} y\right\|_{R^{k+1}}$.

3. $u_{k}=u_{0}+V_{k} y$.

We can use the fact that

$$
r_{k}=f-A u_{k}=V_{k+1}\left(\beta e_{1}+H_{k} y\right)=r_{0}+V_{k+1} H_{k} y
$$

to recover $\bar{u}_{k}$ with no additional operator-vector products involving $A$. In fact if $z=H_{k} y$ and we define a vector

$$
w=\left(w_{1}, w_{2}, \ldots, w_{k+1}\right)^{T} \in R^{k+1}
$$

by $w_{i}=z_{i}+y_{i}$ for $1 \leq i \leq k$ and $w_{k+1}=z_{k+1}$, we have

$$
\bar{u}_{k}=\bar{u}_{0}+V_{k+1} w
$$

which can simply replace the computation of $u_{k}$ in step 3 of gmres. We will refer to the resulting algorithm as smoothed GMRES. Note that smoothed GMRES differs from GMRES only in the final output, where (2.3) is used to compute the final result.

As an algorithm for solution of linear compact fixed point problems, smoothed GMRES shares two properties with Broyden's method. Both converge superlinearly to the solution in the topologies of both $H$ and $X$ and both require storage of the iteration history. Standard implementations of Broyden's method require storage of two vectors for each iteration, [4], [13], [3]. A recent paper [8] reduces that requirement to a single vector, making the two algorithms competitive. In $\S 3$ we present some numerical experiments comparing smoothed GMRES, GMRES, and Broyden's method.

The algorithm in $[8]$ is directed at nonlinear problems. We close this section with an algorithmic description of an implementation targeted at linear equations.

Algorithm 2.2. Algorithm broyden_lin $(x, A, b, \epsilon)$

1. $s=r=f-A u, w_{-1}=s /\|s\|_{H}, C_{-1}=1, \rho=\|r\|_{H}$

2. For $k=0, \ldots$
(a) $u=u+s$
(b) Evaluate $r=f-A u, \rho=\|r\|_{H}$
if $\rho \leq \epsilon\|b\|$ terminate
(c) $w_{k}=-r /\|s\|_{H}$
(d) for $j=0, \ldots, k-1$
$w_{k}=w_{k}-C_{j-1} w_{j}\left(w_{j-1}, w_{k}\right)$
(e) $w_{k}=\left.w_{k}|| s\right|_{H} /\left(1+\left(s, w_{k}\right)\right)$
(f) $s=-\|s\|_{H} w_{k}$
(g) $C_{k}=-1 /\left.|| w_{k}\right|_{H}$

3. Example. We consider the integral operator on $H=L^{2}[0,1]$ given by

$$
K u(x)=\int_{0}^{1} k(x, y) u(y) d y
$$

where

$$
k(x, y)=100\left(\frac{x+5 y}{2+y-x}\right)^{3 / 2} \sqrt{y}
$$


With this choice of $K, X=C^{2}[0,1]$ and $H=L^{2}[0,1]$. We discretize $K$ by the composite midpoint rule. Hence, if we take $m$ subintervals $I_{i}^{m}=((i-1) / m, i / m)$ for $1 \leq i \leq m$ then the quadrature nodes are $x_{i}^{m}=(i-.5) / m$ and the weights are $h=1 / m$.

We performed three sets of computations, one for each $m=100,200,400$. We constructed $f$ so that the solution $u^{*}$ agreed with $\cos (10 x)$ at the nodal points. We used the initial iterate $u_{0}=0$ and solved the equation with GMRES, smoothed GMRES, and Broyden's method. We terminated the iteration when the discrete $L^{2}$ residual had been reduced by a factor of $\tau=10 / \mathrm{m}^{2}$.

For $m=100$ (Figure 3.1), $m=200$ (Figure 3.2), and $m=400$ (Figure 3.3), we plot relative residual norms as functions of the iteration number in both the discrete $C^{2}$-norm (solid line) and the discrete $L^{2}$-norm (dashed line). In each figure there are plots of residual histories for both GMRES and smoothed GMRES, in those plots the dashed line is the residual curve for GMRES and is the same in both plots. The solid line is the residual norm in $C^{2}$, which we computed directly in GMRES by explicitly computing the residual and its $C^{2}$ norm or by using (2.3). We also plot the errors for the final result as functions of $x \in[0,1]$ for smoothed (dashed line) and unsmoothed (solid line) GMRES.

The computations illustrate how the smoothed GMRES iteration gives better performance in the $C^{2}$ norm. The curves for the various values of $m$ are quite similar, indicating that the infinite dimensional analysis can be observed numerically. The plots of the errors also demonstrate the smoothing effects fo (2.3). Note also that the $C^{2}$ and $L^{2}$ relative residuals for the Broyden iteration, which requires more work that GMRES, are very close, in line with the theory in [7].

The tables were created with MATLAB version 4.0a on a Sun SPARC 1+ workstation running SUN OS 4.1. 
FIG. 3.1. $m=100$
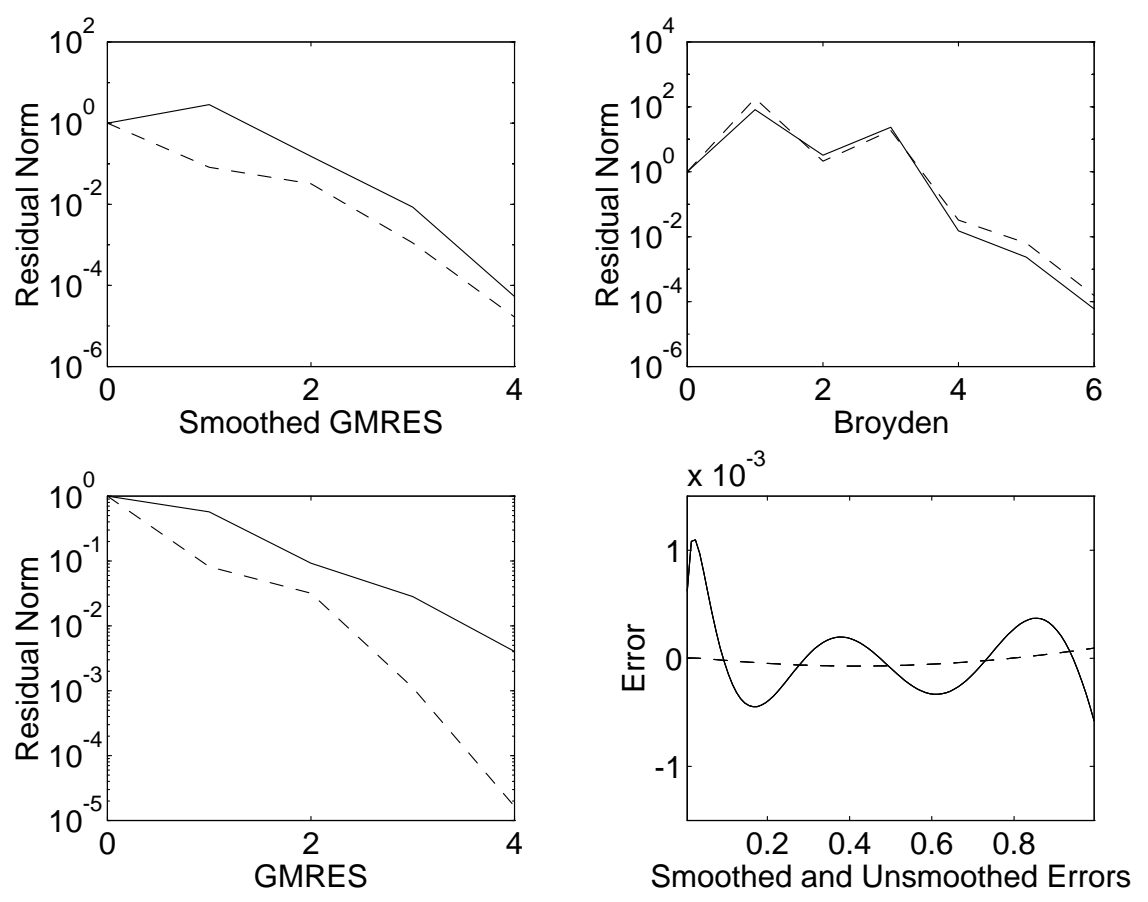
FIG. 3.2. $m=200$
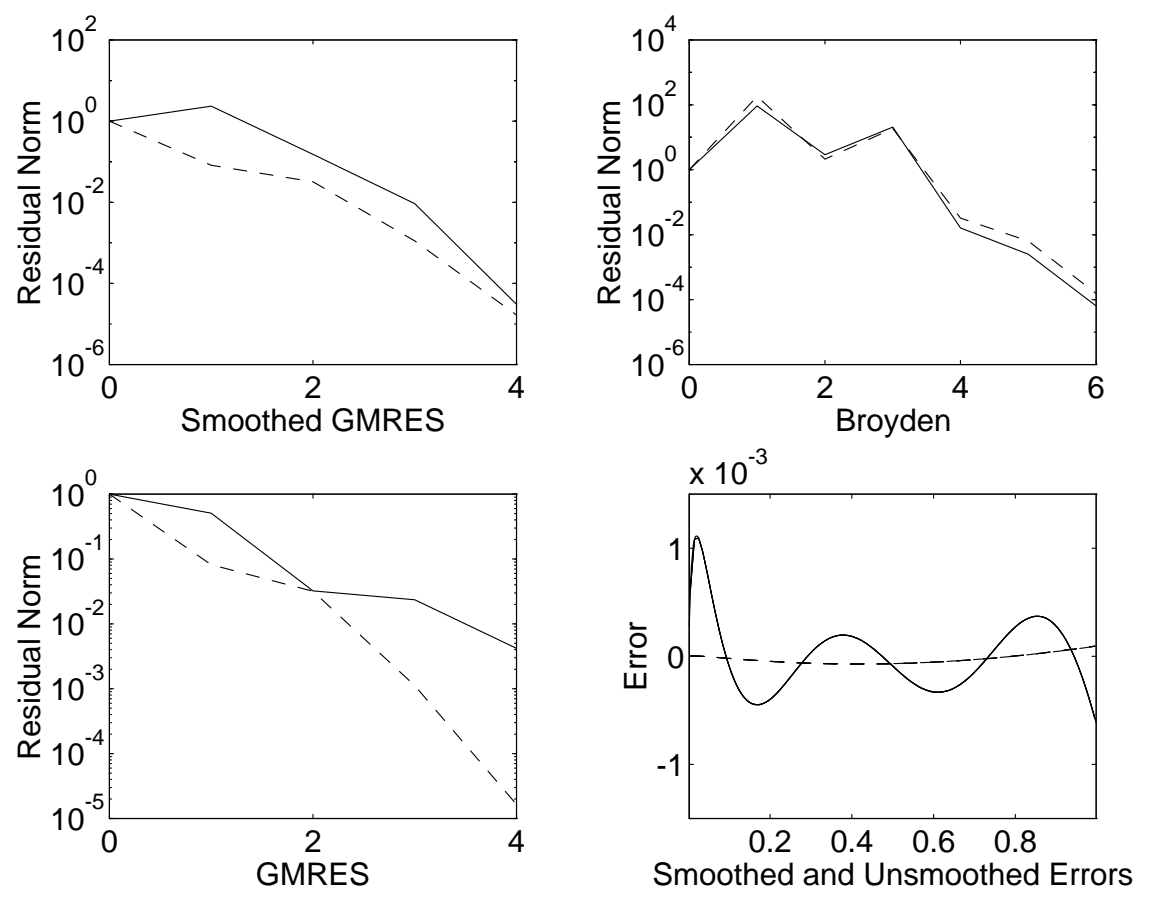
FIG. 3.3. $m=400$
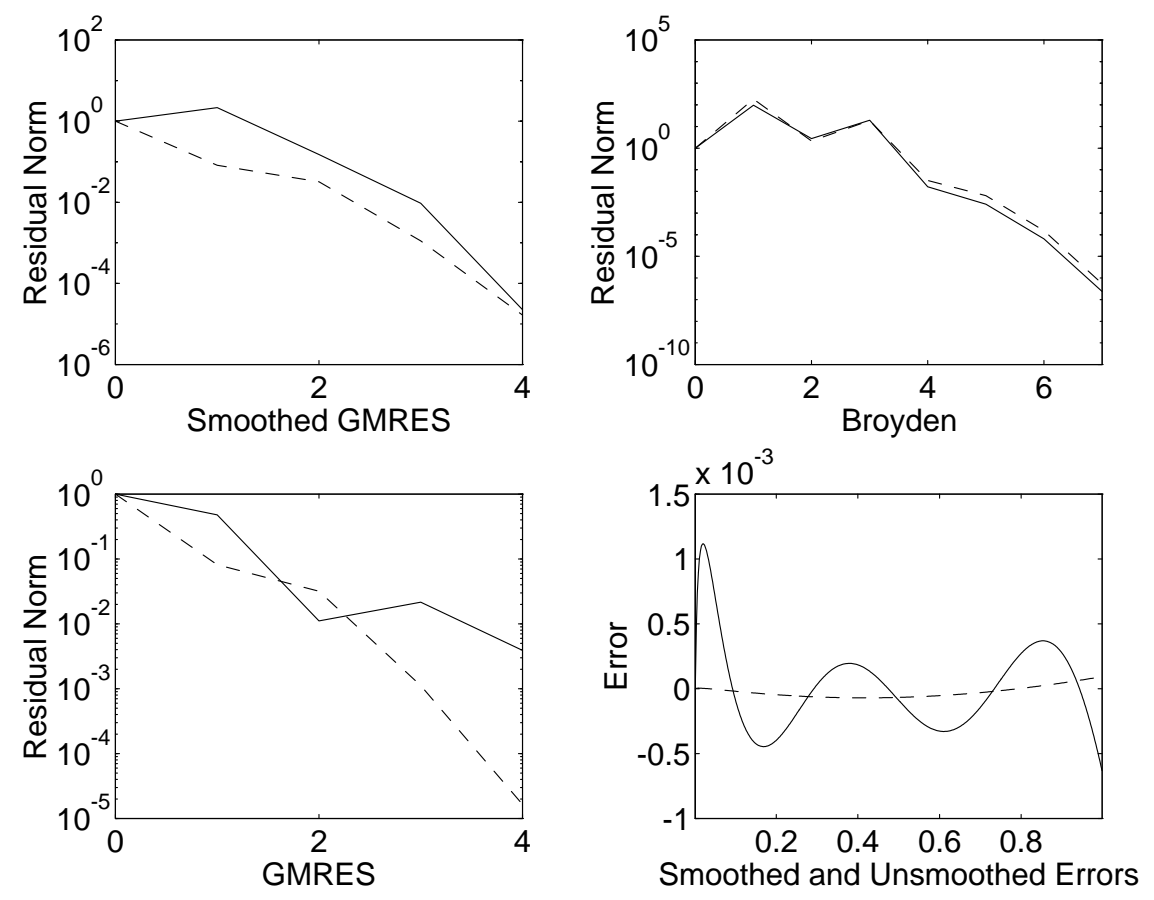
Acknowledgements. The authors thank Anne Greenbaum for the pointer to reference [12].

\section{REFERENCES}

[1] C. G. Broyden, A class of methods for solving simultaneous equations, Math. Comp., 19 (1965), pp. 577-593.

[2] W. Burmeister, Zur Konvergenz einiger verfahren der konjugierten Richtungen, in Proceedings of Internationaler Kongreß über Anwendung der Mathematik in dem Ingenieurwissenschaften, Weimar, 1975.

[3] R. H. Byrd, J. Nocedal, And R. B. Schnabel, Representation of quasi-newton matrices and their use in limited memory methods, Mathematical Programming, 63 (1994), pp. 129-156.

[4] M. Engelman, G. Strang, And K. J. BatHe, The application of quasi-Newton methods in fluid mechanics, International J. for Numerical Methods in Engineering, 17 (1981), pp. 707-718.

[5] D. M. GAY, Some convergence properties of Broyden's method, SIAM J. Numer. Anal., 16 (1979), pp. 623-630.

[6] A. GRIEwank, On the iterative solution of differential and integral equations using secant updating techniques, in The State of the Art in Numerical Analysis, A. Iserles and M. Powell, eds., Oxford, April, 1984 1987, IMA/SIAM, Clarendon Press, pp. 299-324.

[7] D. M. Hwang and C. T. Kelley, Convergence of Broyden's method in Banach spaces, SIAM J. on Optimization, 2 (1992), pp. 505-532.

[8] C. T. Kelley, A new incremental storage implementation of Broyden's method. Submitted for publication.

[9] T. KeRKHOVEN AND Y. SAAD, On acceleration methods for coupled nonlinear elliptic systems, Numerische Mathematik, 60 (1992), pp. 525-548.

[10] J. J. Moré and J. A. Trangenstein, On the global convergence of Broyden's method, Math. Comp., 30 (1976), pp. 523-540.

[11] N. M. Nachtigal, S. C. Reddy, and L. N. Trefethen, How fast are nonsymmetric matrix iterations, SIAM J. Matrix Anal. Appl., 13 (1992), pp. 778-795.

[12] O. Nevanlinna, Convergence of Iterations for Linear Equations, Birkhäuser, Basel, 1993.

[13] J. NocEDaL, Theory of algorithms for unconstrained optimization, Acta Numerica, 1 (1991), pp. 199-242.

[14] E. J. Nyström, Über die praktische Auflösung von Integralgleichungen mit Anwendungen auf Randwertaufgaben, Acta Math., 54 (1930), pp. 185-204.

[15] Y. SAAD AND M. SCHULTZ, GMRES a generalized minimal residual algorithm for solving nonsymmetric linear systems, SIAM J. Sci. Stat. Comp., 7 (1986), pp. 856-869. 